\title{
The Relationship Between State-trait Anxiety and Childhood Trauma in Patients with Acute Chest Pain
}

\author{
(1) Bilge Doğan
}

Department of Psychiatry, Aydın Adnan Menderes University Faculty of Medicine, Aydın, Turkey

\begin{abstract}
Aim: In the present study, we examined to investigate whether state and trait anxiety are related to childhood trauma in patients with acute chest pain (ACP). We hypothesized that childhood trauma and/or state-trait anxiety may be predictive for the distinction between non-cardiac (NCCP) and cardiac chest pain (CCP).

Materials and Methods: A diagnostic interview with 102 ACP patients was performed with DSM-IV Structured Clinical Interview-I (SCID-I). The State and Trait Anxiety Scale I-II (STAI I-II), Hamilton Depression Rating Scale (HDRS), and the short form of Childhood Trauma Scale (CTS-SF) were used to determine the level of state-trait anxiety, depression, and childhood trauma, respectively. The patients with NCCP $(n=63)$ and CPP $(n=39)$ were compared in terms of several sociodemographic and clinical variables. In addition to the correlation analyses, logistic regression analyses were performed to determine the associations of trait anxiety, and origin of cardiac pain.

Results: The rate of male patients tended to be higher in the CCP group compared to the NCCP group ( $p=0.06)$. The patients in the NCPP group were younger than those in the CCP group $(p<0.0001)$. In total sampling, the STAI II scores were significantly correlated with total, physical, and emotional abuse subscale scores of CTQ-SF and with the scores of HDRS. Logistic regression analyses indicated that female gender and earlier age were associated with NCCP.
\end{abstract}

Conclusion: In our study, higher levels of trait anxiety seemed to be correlated with total, emotional, and physical subscale scores of CTQSF. Earlier age and female gender were found to be the predictors for non-cardiac origin of the chest pain.

Keywords: Chest pain, childhood trauma, state -trait anxiety

\section{Introduction}

One of the most frequent reasons for application to emergency departments (ED) is acute chest pain (ACP). In $40-60 \%$ of patients with ACP, a diagnosis of non-cardiac chest pain (NCCP) that excludes angina is considered by cardiologists $(1,2)$. NCCP patients are found to report somatization, anxiety, depression, and decrease in the quality of daily life (3-10). Anxiety has a key role in pain regulation, and some of the autonomic symptoms of anxiety are also seen in NCCP, which may explain the association between higher levels of anxiety and NCCP (11). Epidemiological studies have clearly indicated that adverse childhood experiences, such as abuse or neglect, increase the risk of depression (12), posttraumatic stress disorder (13), panic disorder (14), social anxiety disorder (15), and obsessive-compulsive disorder (16). Previous studies also reported a strong relationship between childhood history of trauma and chronic fatigue syndrome (17), cardiovascular disease (18), fibromyalgia and other chronic pain syndromes $(19,20)$.

Although many authors have studied the psychiatric status of patients with NCCP and ACP separately, there are a few studies investigating the psychiatric profiles of CCP and NCCP patients concurrently. The role of childhood trauma and anxiety for cardiac pain has not been studied enough to date yet. In the present study, we examined the relationship of state and trait anxiety with childhood trauma among a sample of patients with ACP. We hypothesized that adverse childhood experiences were 
associated with higher state and/or trait anxiety in ACP patients. We also supposed that childhood trauma history in relation to state and/or trait anxiety might differentiate the CCP and NCCP patients.

\section{Materials and Methods}

\section{Study Subjects}

G power 3.1.9.2 analysis was used to determine the sample size. We estimated that at least 104 patients should be included in the study (effect size $d=0.493,1$ - $\beta$ err prob $=0.80 ; \alpha$ err prob $=0.05$ ).

We screened 116 patients presenting with ACP, who were admitted to ED of a university hospital between August 2017 and September 2018. The subjects with a known coronary heart disease or other life threatening non-cardiac pathology, (pneumonia, acute abdomen, urinary tract infection), acute ST elevation, hypertensive crisis and a history of psychoactive drug use, a mental disorder like schizophrenia, other psychotic disorders, bipolar affective disorder, and mental retardation were excluded from the study $(n=12)$. The remaining participants ( $n=102$ ) underwent a 4-6-hour observation period by emergency physicians, during which serial physical examination, electrocardiogram, and cardiac enzyme measurements were performed on the arrival and at the sixth hour of admission. All subjects were consulted to the cardiology department for the assessment of a cardiac origin pain. According to the guidelines and current practice in ED for chest pain (21), patients were considered to have low likelihood of being included in the ischemic chest pain group ( $n=63)$ if they had: 1) A judgement of non-angina chest pain made by screening physicians, 2) A normal standard 12-lead ECG with no consecutive change in comparison with previous ECG and, 3) normal cardiac enzyme levels on arrival and six hours later. If all criteria were met, they were recognized as non-cardiac chest pain.

\section{Questionnaire}

After all medical assessments were completed, the patients with CCP $(n=39)$ and NCCP $(n=63)$ were invited to an interview with a psychiatrist provided that their medical status were stable and appropriate for the interview. After obtaining informed consent, participants were given some questionnaires to complete in the emergency clinic after the interview with psychiatrist (B.D), based on the structured clinical interview for DSM IV Structured Clinical Interview-I for Axis I Disorders (SCID-I) (22,23). All the participants were administered the State-Trait Anxiety Inventory (STAI I-II) $(24,25)$ and Hamilton Depression Rating Scale (HDRS) $(26,27)$. STAI I-II is a commonly used scale to measure the severity of state and trait anxiety. It can be used in clinical settings to differentiate between the temporary condition of "state anxiety" and the long-standing "trait anxiety". It has 20 items for measuring trait anxiety and 20 items for state anxiety. Higher scores indicate greater anxiety. A cut-off value of 41 for the STAI-I and a value of 44 for the STAI-II were used to determine the patients with low and high state-trait anxiety. The HDRS is the most widely used clinician-administered depression assessment scale. The original version contains 17 items evaluating the symptoms of depression within the past week. A score of $0-7$ is generally accepted to be within the normal range or in clinical remission.

To assess experienced maltreatment in childhood and adolescence, we used the Short Form of Childhood Trauma Questionnaire (CTQ-SF) $(28,29)$. The scale includes the subscales of emotional abuse (EA), physical abuse (PA), sexual abuse (SA), emotional neglect (EN) and physical neglect (PN). The study received full ethical approval from the University Research Ethics Committee, in accordance with the Helsinki Declaration.

\section{Statistical Analysis}

Data were analyzed on SPSS (Statistical Package of Social Sciences) version 20. Results are presented as mean and standard deviation for the quantitative variables and percentages for the qualitative variables. In the Univariate analysis, the chi-square test was used for the qualitative variables and the Fischer exact test was used wherever applicable. For normally distributed data, the Student's $t$ test was used in comparison of the mean values of two different groups. The relationships among several clinical variables were assessed by the Pearson's correlation analysis.

We performed logistic regression analysis to determine the variables which would predict the distinction between NCCP and CCP. The independent variables were assigned according to the results of the comparison and correlation analyses. Overall percent correct classifications were $72.0 \%$. The possible variables that predicted the severity of trait anxiety were determined through the linear regression analyses.

\section{Results}

Table 1 demonstrates the comparison results of ACP patients with NCCP $(n=63)$ and CCP $(n=39)$. There were no significant differences between two groups in terms of the scores of HDRS and STAI I-II; the total and subscale scores of CTQ-SF. Although the difference did not reach to a significance level, the rate of the males tended to be higher in the CCP group compared to the NCCP group $(p=0.06)$.

In total sample, there was a significant correlation between the STAI-I and STAI-II scores $(r=0.53, p<0.0001)$. As indicated in Table 2 , the STAI-I scores did not correlate with age, the severity of depression, and the total or subscale scores of CTQ-SF. We found 
Table 1. The comparison of cardiac and non-cardiac chest pain patients

\begin{tabular}{|c|c|c|c|c|c|c|c|}
\hline & \multicolumn{2}{|c|}{$\mathrm{CPP}(\mathrm{n}=39)$} & \multicolumn{2}{|c|}{$\operatorname{NCCP}(n=63)$} & \multicolumn{2}{|c|}{ Statistical analyses } & \multirow[b]{2}{*}{$p$} \\
\hline & $\mathbf{n}$ & $\%$ & $\mathbf{n}$ & $\%$ & $\mathrm{X}^{2}$ & df & \\
\hline \multicolumn{8}{|l|}{ Gender } \\
\hline \multirow[t]{2}{*}{ Female } & 12 & 30.8 & 31 & 49.2 & & & \\
\hline & $\mathrm{M}$ & SS & $M$ & SS & $\mathrm{t}$ & $\mathrm{df}$ & $p$ \\
\hline Age & 61.0 & 11.5 & 44.9 & 16.6 & 5.22 & 100 & $<0.0001^{*}$ \\
\hline STAI I & 40.5 & 10.3 & 41.8 & 9.7 & -0.61 & 100 & 0.53 \\
\hline STAI II & 46.5 & 9.6 & 46.0 & 9.7 & 0.22 & 100 & 0.88 \\
\hline CTQ-SF total & 38.2 & 9.37 & 37.9 & 8.9 & 0.17 & 100 & 0.82 \\
\hline Emotional abuse & 6.8 & 2.6 & 7.3 & 2.9 & -0.94 & 100 & 0.34 \\
\hline Physical abuse & 5.4 & 1.23 & 5.7 & 1.8 & -0.71 & 100 & 0.47 \\
\hline
\end{tabular}

${ }^{*} \mathrm{p}<0.05$

CPP: Cardiac chest pain, NCCP: Non-cardiac chest pain, HDRS: Hamilton Depression Rating Scale, STAl: State and Trait Anxiety Scale, CTQ-SF: Short Form of Childhood Trauma Questionnaire

Table 2. The correlations between STAI I-II and several variables

\begin{tabular}{lll}
\hline & STAI-I & STAI-II \\
\hline Age & $r=-0.11, p=0.23$ & $r=-0.12, p=0.20$ \\
\hline HDRS & $r=0.07, p=0.46$ & $r=0.35, p<0.0001^{*}$ \\
\hline CTQ-SF total & $r=0.09, p=0.34$ & $r=0.238, p=0.01^{*}$ \\
Emotional abuse & $r=0.09, p=0.37$ & $r=0.29, p=0.003^{*}$ \\
Physical abuse & $r=0.04, p=0.64$ & $r=0.29, p=0.01^{*}$ \\
Emotional neglect & $r=0.10, p=0.27$ & $r=0.12, p=0.22$ \\
Physical neglect & $r=-0.01, p=0.94$ & $r=0.05, p=0.55$ \\
Sexual abuse & $r=0.10, p=0.30$ & $r=0.13, p=0.19$ \\
\hline * $p<0.05$ & & \\
HDRS: Hamilton Depression Rating Scale, STAl: State and Trait Anxiety Scale, CTQ-SF: \\
Short Form of Childhood Trauma Questionnaire
\end{tabular}

that STAI-II scores were significantly correlated with total $(r=0.23$, $p=0.01)$, physical abuse $(r=0.29, p=0.01)$, and emotional abuse $(r=0.29, p=0.003)$ subscale scores of CTQ-SF, and with the scores of HDRS $(r=0.35, p<0.0001)$.

We performed a logistic regression analysis to determine the variables which would predict the origin of the chest pain. When age, gender, and the scores of HDRS, STAI I-II, and CTQSF were entered into the logistic regression analyses, we found that younger age (OR: 0.92, $\mathrm{Cl}: 0.88-0.95, \mathrm{p}<0.0001)$ and female gender (OR: 3.43, $\mathrm{Cl}: 1.14-10.31, \mathrm{p}=0.02$ ) significantly predicted the patients with NCCP (Table 3).

\section{Discussion}

The relation between psychosocial stress factors and cardiovascular diseases has been an important research topic. There are many studies reporting that anxiety accompanies
Table 3. Multinomial logistic regression for determining the factors which would predict the origin of the chest pain

\begin{tabular}{lllll}
\hline & Beta & OR & $\mathbf{( 9 5 \% ~ C I )}$ & $\mathbf{p}$ \\
\hline Age & -0.081 & 0.922 & $0.889-0.957$ & $<0.0001$ \\
\hline Gender & 1.136 & 3.113 & $1.124-8.621$ & 0.02 \\
\hline HDRS & 0.023 & 1.023 & $0.979-1.069$ & 0.31 \\
\hline STAI & & & & \\
I & 0.020 & 0.952 & $0.963-1.081$ & 0.49 \\
II & 0.039 & 0.962 & $0.910-1.017$ & 0.17 \\
\hline CTQ-SF & 0.008 & 1.008 & $0.947-1.073$ & 0.80 \\
\hline${ }^{*}$ p $<0.05$ & & & &
\end{tabular}

HDRS: Hamilton Depression Rating Scale, STAI: State and Trait Anxiety Scale, CTQ-SF: Short Form of Childhood Trauma Questionnaire, Cl: Confidence interval

chest pain in 31-56\% of patients. Anxiety -related disorders are often considered among the causes for NCCP $(30,31)$. Some of the previous studies reported that patients with NCCP were found to be more worried, tense and frightened when compared to the reference group (8). Anxiety and emotional stress may lead to chest pain by causing increased muscle tension (32) or chronic activation of hypothalamic-pituitary-adrenal axis (33). In addition, the prevalences rates of anxiety and depression were found to be similar among individuals with a cardiac diagnosis and those diagnosed with NCCP $(34,35)$. In accordance with these reports, our findings demonstrated that there were no significant differences in STAI I-II, HDRS, and CTQ-SF scores between ACP patients with NCCP and CCP. However, we have found a strong relationship between the severities of state and trait anxiety in total sample. In contrast to state anxiety scores, the severity of depression, and emotional and physical abuse appeared to be 
strongly correlated with trait anxiety scores. Particularly, physical abuse was found to significantly predict the severity of trait anxiety. Therefore, these results may suggest that the subjects who experienced more severe childhood trauma had more anxious traits and became more susceptible to develop an anxiety in case of ACP. The patients with higher anxious traits also appeared to be more depressive during ACP. Previously, high levels of trait anxiety have been reported in adult and adolescent patients with histories of childhood physical and sexual abuse $(36,37)$. However, our findings might not indicate that the relationship between childhood trauma and anxiety scores is specific for ACP patients. These results should be confirmed in larger samples in comparison to healthy controls or to patients with other somatic complaints.

Exposure to childhood maltreatment and psychological trauma has been associated with increased vulnerability to adult psychiatric and physical health outcomes. Abuse survivors also report experiencing more pain in relation to their health problems $(38,39)$. It is known that the levels of anxiety and depression are high in these patients, and chronic pain is related to the somatization of anxiety and depression (39). Several theories have been proposed to explain the increased pain in trauma sufferers. Childhood abuse is associated with a negative attributional style (37) and an inability to manage stress (40) to exacerbate pain. Trauma-related alterations may amplify pain by lowering pain thresholds (41). In this study, we hypothesized that childhood trauma might be a clinical marker which would help to differentiate the CCP and NCPP. In contrary to our hypothesis, we failed to find any associations between the origin of chest pain and the childhood trauma or state-trait anxiety in patients with ACP. Female gender and the earlier ages of the patients appeared to be the strongest predictors of NCCP. In consistent with our findings, some of the previous studies reported that female patients with chest pain presented with anxiety more frequently (42). Thus, female patients with chest pain are twice more consulted to cardiologists when compared to male patients (43). While male patients display psychological symptoms of anxiety such as tension and an intuition of a bad event, female patients tend to present the physical symptoms of anxiety including chest pain, palpitation, shortness of breath, and nausea (44).

\section{Conclusion}

We suggest that childhood trauma, particularly physical abuse may be associated with trait anxiety, and therefore may predispose the patients to develop an acute anxiety during ACP. In contrast to our expectations, adverse experiences in childhood did not seem to be related to the type of chest pain. Emergency physicians should evaluate patients according to clinical and laboratory findings rather than subjective criteria such as level of anxiety. The age and gender were prominent predictors of a distinction between CCP and NCCP. These findings should be confirmed by further investigations with larger groups of patients. The major limitation of this study was the relatively smaller size of the sample. We did not assess the frequency of traumatic experiences, duration of the trauma, and the age when trauma first occurred. We could not examine the relationship of the severity of childhood trauma, and anxiety with chest pain.

\section{Ethics}

Ethics Committee Approval: Aydın Adnan Menderes University (approval number: 2016/878).

Informed Consent: It was taken.

Peer-review: Externally and internally peer-reviewed.

Financial Disclosure: The author declared that this study received no financial support.

\section{References}

1. Eken C, Oktay C, Bacanli A, Gulen B, Koparan C, Ugras SS, et al. Anxiety and depressive disorders in patients presenting with chest pain to the emergency department: A comparison between cardiac and non-cardiac origin. J Emerg Med 2010;39:144-50.

2. Mayou RA, Thompson DR. Treatment needs of patients admitted for acute chest pain. J Psychosom Res 2002;53:1177-83.

3. Hocaoglu C, Gulec MY, Durmus I. Psychiatric comorbidity in patients with chest pain without a cardiac etiology. Isr J Psychiatry Relat Sci 2008;45:49-54.

4. García-Campayo J, Rosel F, Serrano P, Santed MA, Andres E, Roca M, et al. Different psychological profiles in non-cardiac chest pain and coronary artery disease: a controlled study. Rev Esp Cardiol 2010;63:357-61.

5. Husser D, Bollmann A, Kühne C, Molling J, Klein HU. Evaluation of noncardiac chest pain: diagnostic approach, coping strategies and quality of life. Eur J Pain 2006;10:51-5

6. Eslick GD, Talley NJ. Natural history and predictors of outcome for noncardiac chest pain: a prospective 4-year cohort study. Neurogastroenterol \& Motility 2008;20:989-97.

7. Hadlandsmyth K, Rosenbaum DL, Craft JM, Gervino EV, White KS. Health care utilisation in patients with non-cardiac chest pain: A longitudinal analysis of chest pain, anxiety and interoceptive fear. Psychol Health 2013;28:849-61.

8. Smeijers L, van de Pas H, Nyklicek I, Notten PJ, Pedersen SS, Kop WJ. The independent association of anxiety with non-cardiac chest pain. Psychol Health 2014;29:253-63.

9. Webster R, Norman P, Goodacre S, Thompson A. The prevalence and correlates of psychological outcomes in patients with acute non-cardiac chest pain: a systematic review. Emerg Med J 2012;29:267-73.

10. Webster R, Thompson AR, Norman P. "Everything's fine, so why does it happen?" A qualitative investigation of patients' perceptions of noncardiac chest pain. J Clin Nurs 2015;24:1936-45.

11. Huffman JC, Pollack MH. Predicting panic disorder among patients with chest pain: an analysis of the literature. Psychosomatics 2003;44:222-36.

12. Widom CS, DuMont K, Czaja SJ. A prospective investigation of major depressive disorder and comorbidity in abused and neglected children grown up. Arch Gen Psychiatry 2007;64:49-56. 
13. Widom CS. Posttraumatic stress disorder in abused and neglected children grown up. Am J Psychiatry 1999;156:1223-9.

14. David D, Giron A, Mellman TA. Panic-phobic patients and developmental trauma. J Clin Psychiatry 1995;56:113-7.

15. Chartier MJ, Walker JR, Stein MB. Social phobia and potential childhood risk factors in a community sample. Psychol Med 2001;31:307-15.

16. Mathews CA, Kaur N, Stein MB. Childhood trauma and obsessive-compulsive symptoms. Depress Anxiety 2008;25:742-51.

17. Heim C, Wagner D, Maloney E, Papanicolaou DA, Solomon L, Jones JF, et al. Early adverse experience and risk for chronic fatigue syndrome: results from a population-based study. Arch Gen Psychiatry 2006;63:1258-66.

18. Imbierowicz K, Egle UT. Childhood adversities in patients with fibromyalgia and somatoform pain disorder. Eur J Pain 2003;7:113-9.

19. Heim C, Ehlert U, Hanker JP, Hellhammer DH. Abuse-related posttraumatic stress disorder and alterations of the hypothalamic-pituitary-adrenal axis in women with chronic pelvic pain. Psychosom Med 1998;60:309-18.

20. Romans S, Belaise C, Martin J, Morris E, Raffi A. Childhood abuse and later medical disorders in women. An epidemiological study. Psychother Psychosom 2002;71:141-50.

21. Pollack CV, Roe MT, Peterson ED. 2002 update to the ACC/AHA guidelines for the management of patients with unstable angina and non-ST-segment elevation myocardial infarction: Implications for emergency department practice. Ann Emerg Med 2003;41:355-69.

22. First M, Williams Janet BW, Spitzer R, Gibbon M. Structured clinical interview for DSM-IV clinical version (SCID-I/CV). Washington DC: American Psychiatric Press, 1997.

23. Ozkurkcugil A, Aydemir O, Yıldız M, Danacı AE. The Turkish reliability and validity study of Structured clinical interview for DSM-IV clinical version (SCID-I/CV). J Drug Treat 1999; 12(4): 233-36.

24. Spielberger CD, Gorsuch RL, Lushene R, Jacobs AG. Manual for the State-Trait Anxiety Inventory. Palo Alto, CA: Consulting Psychologists Press, 1983.

25. Oner N, LeCompte A. State-Trait Inventory, Handbook, Bosphorus University Press, 1985.

26. Hamilton M. A rating scale for depression. J Neurol Neurosurg Psychiatry 1960;23:56-62.

27. Akdemir A, Türkçapar MH, Orsel SD, Demirergi N, Dag I, Ozbay MH. Reliability and validity of the Turkish version of the Hamilton Depression Rating Scale. Compr Psychiatry 2001;42:161-5.

28. Sar V, Öztürk E, Ikikardes E. Validity and reliability of the Turkish Version of Childhood Trauma Questionnaire. J Med Sci 2012;32:1054-63.

29. Bernstein DP, Stein JA, Newcomb MD, Walker E, Pogge D, Ahluvalia T, et al. Development and validation of a brief screening version of the Childhood Trauma Questionnaire. Child Abuse and Negl 2003;27:169-90.
30. White KS, Craft JM, Gervino EV. Anxiety and hypervigilance to cardiopulmonary sensations in non-cardiac chest pain patients with and without psychiatric disorders. Behav Res Ther 2010;48:394-401.

31. Jonsbu E, Dammen T, Morken G, Lied A, Vik-Mo H, Martinsen EW. Cardiac and psychiatric diagnoses among patients referred for chest pain and palpitations. Scand Cardiovasc J 2009;43:256-9.

32. Lundberg U, Dohns IE, Melin B, Sandsjö L, Palmerud G, Kadefors R, et al. Psychophysiological stress responses, muscle tension, and neck and shoulder pain among supermarket cashiers. J Occup Health Psychol 1999;4:245-55.

33. Fries E, Hesse J, Hellhammer J, Hellhammer DH. A new view on hypocortisolism. Psychoneuroendocrinology 2005;30:1010-6.

34. Chambers JB, Marks EM, Hunter MS. The head says yes but the heart says no: what is non-cardiac chest pain and how is it managed? Heart 2015;101:12409.

35. Gümüșer F, Altinbaș K, Çăglar IM, Ungan İ. Comparison of temperamental features, anxiety, and depression levels between non-cardiac angina and acute coronary syndrome. Noro Psikiyatr Ars 2014;51:363-7.

36. Handa M, Nukina H, Hosoi M, Kubo C. Childhood physical abuse in outpatients with psychosomatic symptoms. Biopsychosoc Med 2008;21:2-8.

37. Martin L, Viljoen M, Kidd M, Seedat S. Are childhood trauma exposures predictive of anxiety in school attending youth? J Affect Disord 2014;168;512.

38. Sachs-Ericsson N, Verona E, Joiner T, Preacher KJ. Parental verbal abuse and the mediating role of self-criticism in adult internalizing disorders. J Affect Disord 2006;93:71-8.

39. Sachs-Ericsson N, Kendall-Tackett K, Hernandez A. Childhood abuse, chronic pain, and depression in the National Comorbidity Survey. Child Abuse Negl 2007;31:531-47.

40. Tylee A, Gandhi P. The Importance of Somatic Symptoms in Depression in Primary Care. Prim Care Companion J Clin Psychiatry. 2005;7:167-76.

41. Kendall-Tackett K. Chronic pain: the next frontier in child maltreatment research. Child Abuse Negl 2001;25:997-1000.

42. Arnow BA, Hart S, Hayward C, Dea R, Barr Taylor C. Severity of child maltreatment, pain complaints and medical utilization among women. J Psychiatr Res 2000;34:413-21.

43. Grace SL, Abbey SE, Irvine J, Shnek ZM, Stewart DE. Prospective examination of anxiety persistence and its relationship to cardiac symptoms and recurrent events. Psychother Psychosom 2004;73:344-52.

44. Chiaramonte GR, Friend R. Medical students' and residents' gender bias in the diagnosis, treatment and interpretation of coronary heart disease symptoms. Health Psychol 2006;25:255-66.

45. Paykel ES. Life events, social support and depression. Acta Psychiatr Scand Suppl 1994;377:50-8. 